\title{
Modulating the import of medium-chain alkanes in E. coli through tuned expression of FadL
}

\author{
Toby P. Call ${ }^{1,2^{*}}$, M. Kalim Akhtar ${ }^{1,3}$, Frank Baganz $^{1}$ and Chris Grant $^{1}$
}

\begin{abstract}
Background: In recent years, there have been intensive efforts to develop synthetic microbial platforms for the production, biosensing and bio-remediation of fossil fuel constituents such as alkanes. Building predictable engineered systems for these applications will require the ability to tightly control and modulate the rate of import of alkanes into the host cell. The native components responsible for the import of alkanes within these systems have yet to be elucidated. To shed further insights on this, we used the AlkBGT alkane monooxygenase complex from Pseudomonas putida GPo1 as a reporter system for assessing alkane import in Escherichia coli. Two native $E$. coli transporters, FadL and OmpW, were evaluated for octane import given their proven functionality in the uptake of fatty acids along with their structural similarity to the P. putida GPo1 alkane importer, AlkL.

Results: Octane import was removed with deletion of fadL, but was restored by complementation with a fadL-encoding plasmid. Furthermore, tuned overexpression of FadL increased the rate of alkane import by up to 4.5- fold. A FadL deletion strain displayed a small but significant degree of tolerance toward hexane and octane relative to the wild type, while the responsiveness of the well-known alkane biosensor, AlkS, toward octane and decane was strongly reduced by 2.7- and 2.9-fold, respectively.

Conclusions: We unequivocally show for the first time that FadL serves as the major route for medium-chain alkane import in E. coli. The experimental approaches used within this study, which include an enzyme-based reporter system and a fluorescent alkane biosensor for quantification and real-time monitoring of alkane import, could be employed as part of an engineering toolkit for optimizing biological systems that depend on the uptake of alkanes. Thus, the findings will be particularly useful for biological applications such as bioremediation and biomanufacturing.
\end{abstract}

Keywords: Alkanes, Transport proteins, Biosensors, Bio-oxidation, Import, Solvent tolerance

\section{Background}

Fossil fuels including oil, coal and gas still serve as our predominant source of energy, meeting over $85 \%$ of our requirements [1]. Environmental, social and ecological issues have spurred on alternative approaches for fulfilling energy demands. In recent years, intensive efforts have been placed on the development of microbial platforms for the production and waste-management of

\footnotetext{
* Correspondence: tpc38@cam.ac.uk

'Department of Biochemical Engineering, Advanced Centre for Biochemical Engineering, University College London, Torrington Place, London WC1E 7JE, UK

${ }^{2}$ Present address: Department of Biochemistry, University of Cambridge, 80 Tennis Court Road, Cambridge CB2 1GA, UK

Full list of author information is available at the end of the article
}

fossil fuel constituents such as alkanes. Numerous studies have shown that microbes can be engineered for the biosynthesis, bioremediation and biosensing of alkanes [2-5]. An important aspect of developing and optimizing such systems is to understand the transport characteristics of the microbial host. However to date, the passage of alkanes across the membranes still remains a poorly understood area.

AlkL, which forms part of the well-characterized alkane degradation pathway in Pseudomonas putida GPo1, was identified as the first known bacterial alkane importer [6]. We showed previously that heterologous expression of alkL in E. coli could improve dodecane (C12) oxidation by up to 2 orders of magnitude [4]. The uptake process was evaluated by using the $P$. putida 
alkane monooxygenase (AlkB). This inner membrane complex receives electrons from $\mathrm{NADH}$, via rubredoxin reductase (AlkT) and rubredoxin (AlkG), and transfers one oxygen to the alkane substrate while the other is reduced to $\mathrm{H}_{2} \mathrm{O}$ [7]. Ultimately, it oxidizes alkanes to fatty alcohols, fatty aldehydes, and fatty acids and has been investigated industrially for the bioconversion of octane to octanol [8]. In P. putida, alkanes oxidized to fatty acids are gradually converted to acyl-CoA by AlkK (FadD in Escherichia coli) for entry into the $\beta$-oxidation cycle $[7,9]$.

It is known from previous studies that $E$. coli is capable of medium chain alkane import $[10,11]$. We suspected two possible transporters. The first was the OmpW beta barrel outer membrane channel in E. coli, which has $27 \%$ sequence homology to AlkL [6]. The second was the outer membrane protein FadL, which is the principal route for aliphatic fatty acid import $[12,13]$ and shares some structural similarities to AlkL (Fig. 1). All three transporters: AlkL, OmpW, and FadL possess a similar lateral transfer mechanism that allows the entry of small hydrophobic molecules into the outer membrane through an opening in the barrel wall $[4,14,15]$ (Fig. 1a-c). In the case of FadL, sequential binding of long-chain fatty acids (LCFAs) with low- and highaffinity binding domains results in a conformational change of FadL (Fig. 1d). The plug domain is eventually displaced (Fig. 1e), forming a passage through a conserved kink in the $\beta$-sheet wall [16] that allows lateral diffusion of the LCFA into the outer membrane (Fig. 1f).

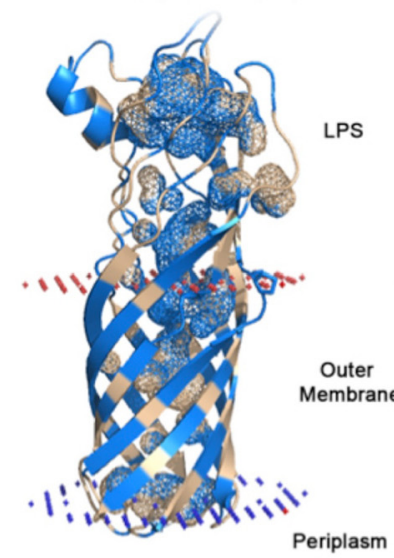

a)

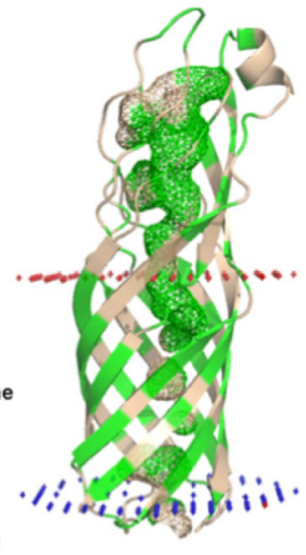

b)

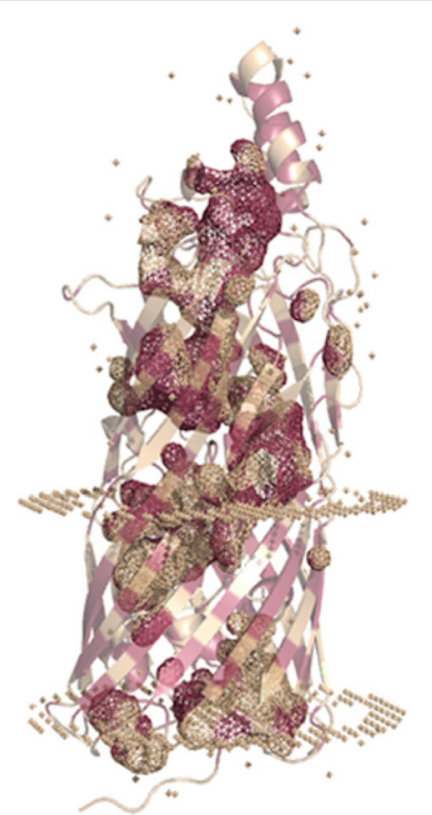

c)

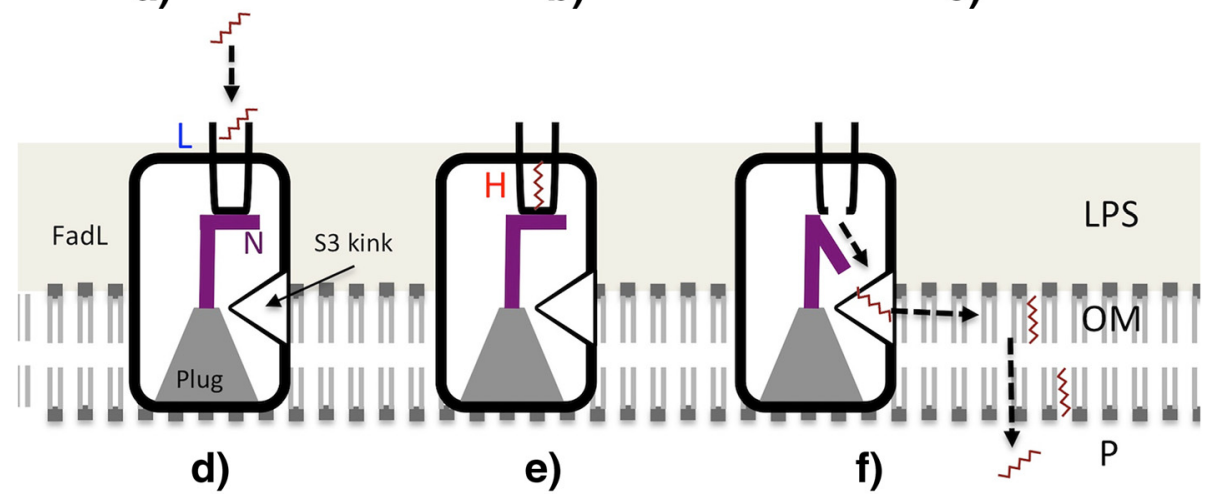

Fig. 1 Cartoon structural representations generated in Pymol showing the internal hydrophobic channels. a AlkL, b OmpW, and c FadL spanning the outer membrane. The coloured areas represent hydrophobic regions, including the internal hydrophobic channels leading to the gap in the $\beta$-sheet wall. In a model for the mechanism of lateral diffusion of small hydrophobic substrates through FadL: $\mathbf{d}$ extracellular substrates, in this case octane, bind sequentially to a low affinity binding site $(\mathrm{L})$; e then a high affinity binding site $(\mathrm{H})$, $\mathbf{f}$ leading to conformational change at the $\mathrm{N}$-terminus (purple) of the plug. This creates a continuous channel through which the substrate can diffuse through to an opening caused by a kink in the $\mathrm{S} 3 \beta$-sheet strand and into the outer membrane 
Within the hydrophobic binding pocket of FadL, the positively charged residues of $\mathrm{Arg}^{157}$ and $\mathrm{Lys}^{317}$ most likely confer specificity to LCFAs by binding to the negatively charged carboxyl head group [17]. Once in the outer membrane, LCFAs are thought to be adsorbed to the inner membrane and 'flip-flop' to its cytosolic face; however the precise route still remains to be elucidated [18].

In this study, we investigated OmpW and FadL as potential transporters for the native import of alkanes in $E$. coli using a range of experimental approaches (Fig. 2). We identified the major route of alkane import in E. coli and highlight the importance of modulating expression levels of membrane transporters for optimal alkane uptake. Furthermore, we show that FadL can significantly alter the responsivity of alkane biosensing in whole-cell systems in addition to improving alkane tolerance. Overall, this study provides a generalizable framework for the evaluation and characterization of alkane import in whole-cell systems.

\section{Results and discussion}

Import of octane in E. coli is mediated by FadL

To determine whether OmpW and FadL transporters might be involved in the native uptake of octane, the $\Delta f a d L$ and $\Delta o m p W$ strains from the Keio E. coli knockout collection [19] were transformed with the plasmid
pGEc41 [8] encoding the alkane inducible alkBGT alkane monooxygenase complex. With this plasmid, cultivation in the presence of $30 \%(\mathrm{v} / \mathrm{v})$ octane leads to the formation of three oxidized products: 1-octanol, 1-octanal and 1-octanoic acid, as reported earlier [4]. The synthesis of 1-octanol and 1-octanal is attributed to AlkB while the synthesis of 1-octanoic acid is catalyzed either by AlkB and/or native enzymes that have yet to be identified.

We had shown previously [4] that octane uptake into the cell was the rate limiting step in this bioconversion. By using an excess of octane substrate to overcome mass-transfer rate limitations, we were able to assess the import of octane based on its conversion to the three aforementioned oxidized products. After $8 \mathrm{~h}$ of cultivation in a sealed deep-well plate, the total level of oxidized products (1-octanol, 1-octanal and 1-octanoic acid) was separated and quantified by gas chromatography. The $\Delta o m p W$ pGEc41 strain $(126 \pm 10 \mathrm{mg}$ oxidized products/l) showed no significant reduction compared to the wild type strain $(129 \pm 40 \mathrm{mg} / \mathrm{l}$ of oxidized products), while greatly reduced levels were observed for the $\Delta f a d L$ counterpart $(0.34 \pm 0.1 \mathrm{mg} / \mathrm{l})$ (Fig. 3). Complementation of $\Delta f a d L$ with a plasmid encoding FadL from the ASKA E. coli ORF collection, pASKAfadL, restored conversion of octane to $88 \pm$ $16 \mathrm{mg} / \mathrm{l}$, providing strong initial evidence that octane import in E. coli proceeds via FadL.

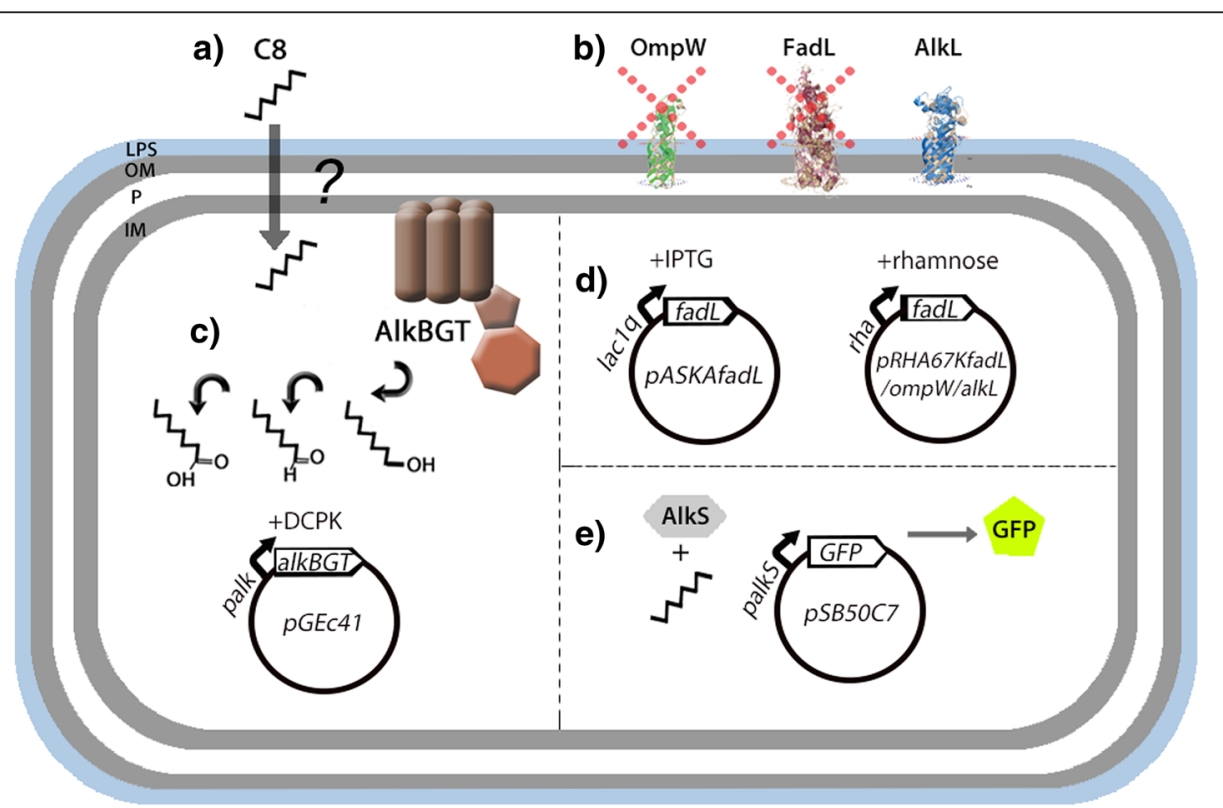

Fig. 2 Model system for evaluating (a) short chain alkane uptake in E. Coli. b Knockout strains for candidate transporters OmpW and FadL were selected from the Keio collection. c The AlkBGT alkane monooxygenase complex from P. putida, encoded on pGEc41, was used as a reporter for alkane import, allowing quantification of intracellular alkane oxidation to alcohols, aldehydes, and fatty acids by gas chromatography. $\mathbf{d}$ Complementation of fadL in the $\triangle f a d L$ knockout strain to restore phenotype was performed with IPTG inducible vector PASKAfadL, whereas the rhamnose inducible vectors: pRHA67KfadL, pRHA67KompW, and pRHA67KalkL permitted fine tuning of overexpression to optimize alkane import via FadL, OmpW, and known alkane importer AlkL. e pSB50C7, harboring an alkane inducible AlkS-GFP fluorescence biosensor, was used as a independent reporter system to confirm the results from AlkBGT alkane oxidation in the $\Delta \mathrm{fadl}$ strain 


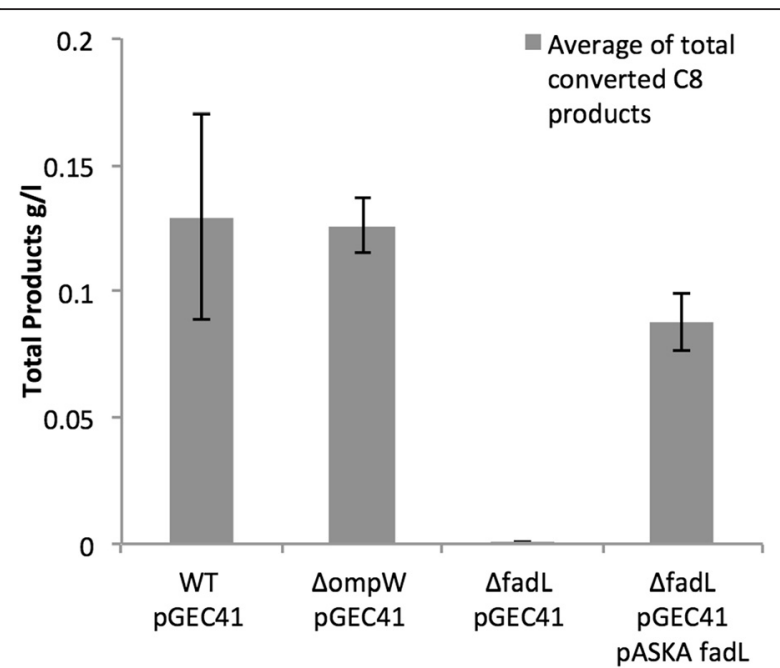

Fig. 3 Impact of E. coli FadL and OmpW mutants on the whole-cell bioconversion of octane. Experiments were performed in BW25113 single deletion mutants and strains complemented with ASKA plasmid encoding for fad $\mathrm{L}$ and ompW. The total levels of oxidized products was determined by GC-FID after $8 \mathrm{~h}$ in partially sealed wells using octane as substrate. Results are an average of at least 3 biological replicates and error bars show the standard error

This has interesting implications in our current understanding of the substrate binding of FadL which has long been known to play a physiological role in the import of long chain fatty acids in E. coli $[12,13]$. Previously, Black et al. [20] had shown that deletion of the His ${ }^{110}$ residue could significantly reduce the rate of fatty acid binding and import, indicating its importance in the interaction of the carboxylic group with FadL. From structural studies, the zwitterionic head group of the detergent molecule, lauryldimethylamine-oxide (LDAO) is positioned close to $\mathrm{Arg}^{157}$ and $\mathrm{Lys}^{317}$ suggesting that these residues are also necessary for carboxylic group interaction [14]. Kinetic studies show an almost 10-fold increase in $\mathrm{K}_{\mathrm{m}}$ for shorter chain fatty acids (C6-C9) compared to LCFAs (C12-C16) [13]. As octane is an aliphatic hydrocarbon without the negatively-charged carboxylic head group, these results do suggest that the binding of substrates to FadL is not necessarily dependent upon the presence of electrostatic charges.

\section{Optimal import of alkanes requires tuned expression of protein transporters}

We noted a complex interplay between IPTG-based membrane transporter overexpression and octane conversion. For example, transforming E. coli HB101 strain with pASKAfadL led to a reduction in the specific activity of octane

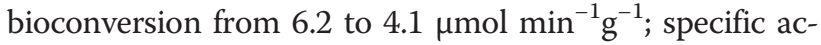
tivity further decreased to 2.1 and then to $0.48 \mu \mathrm{mol}$ min ${ }^{-1} \mathrm{~g}^{-1}$ with induction of recombinant FadL at $50 \mu \mathrm{M}$ and $500 \mu \mathrm{M}$ IPTG respectively (Additional file 1: Figure S1a).
By switching to the $E$. coli Keio $\Delta f a d L$ strain complemented with a pASKAfadL, a slight increase in activity from 3.1 to $3.25 \mu \mathrm{mol} \mathrm{min} \mathrm{g}^{-1}$ between 0 and $50 \mu \mathrm{M}$ IPTG induction was initially observed which drastically decreased to $0.71 \mu \mathrm{mol} \mathrm{min} \mathrm{m}^{-1}$ at the higher concentration of $500 \mu \mathrm{M}$ IPTG (Additional file 1: Figure S1b). Taken together, these observations corroborate our previous findings in which octane conversion was found to be inversely correlated with AlkL induction [4]. Even though the mode of inhibition remains to be proven, these findings strongly support the notion that high expression level of membrane importers may not necessarily offer a sound approach for achieving optimal rates of bioconversion and could, in fact, be detrimental to the physiology of the host organism.

In order to precisely evaluate the importance of transporter induction for alkane import, a multifactorial approach was adopted with two important modifications made to the experimental approach. Firstly, vectors pRHA67KompW, pRHA67KalkL, and pRHA67KfadL were constructed with the transporters ompW, alkL, and fadL, under control of the rhaBAD promoter, previously shown to allow a more tightly controlled expression of the outer membrane protein AlkL [4]. In order to evaluate the performance of the transporters in an industrially relevant context, we tested the genes in the HB101 strain in which we had previously witnessed the highest activity of the alkB enzyme complex. These plasmids were transformed into the wild type HB101 strain, along with pGEc41, and the resulting strains induced with rhamnose concentrations ranging from 0 to $1000 \mu \mathrm{M}$. Secondly, the assay was restricted to a one hour growth period within the exponential phase in order to reduce the likelihood of further converting 1-octanol to unknown metabolites by the host organism. ANOVA was applied to the full data set to determine any statistical significance between the variables and the observed outcomes. Highly significant effects on alkane conversion were found for strains harbouring the recombinant FadL and AlkL transporters though not for the OmpW transporter ( $\mathrm{T}$ ratio of 4.55 and $p=0.0002$ for FadL) (Additional file 1: Figure S2A), as well as a statistically significant cooperative effect between inducer concentration and transporter induction ( $\mathrm{T}$ ratio of 3.46 and $p=0.0023$ for FadL $\times$ Inducer concentration Additional file 1: Figure S2B). Specific activity of FadL peaked at

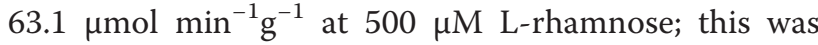
found to be 2.8 -fold higher than OmpW $\left(22.7 \mu \mathrm{mol} \mathrm{min}^{-1} \mathrm{~g}^{-1}\right.$ ) at $10 \mu \mathrm{M}$ L-rhamnose, and 4.5 -fold higher than the wild type at $13.8 \mu \mathrm{mol} \mathrm{min}^{-1} \mathrm{~g}$ ${ }^{-1}$, also treated with L-rhamnose (Fig. 4). Inducing omp W expression caused a slight increase at $10 \mu \mathrm{M}$ followed by a reduction in octane bioconversion at higher L-rhamnose concentrations, similar to the effect seen with FadL and AlkL in the high expression- 


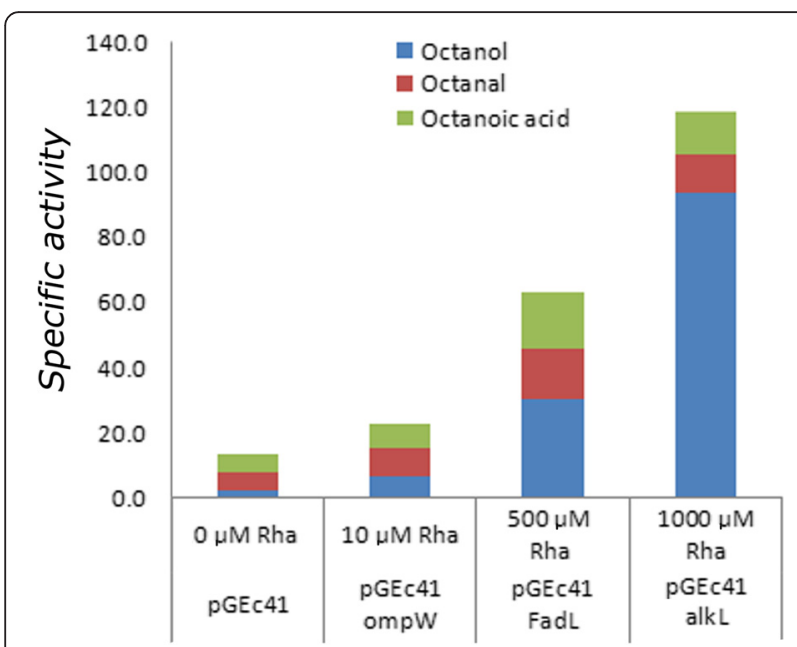

Fig 4 Effect of tightly-regulated expression of E. coli FadL and OmpW on the whole-cell bioconversion of octane. Specific maximum specific activity conditions in single well reactions from a multifactorial inducer study (Additional file 1: Figure S2) after $1 \mathrm{~h}$ overexpression of OmpW, FadL, and AlkL in the E. coli HB101 background strain from an optimized, L-rhamnose inducible vector at optimal inducer concentrations identified for each protein $(10,50,500$, and $1000 \mu \mathrm{M})$, compared with wild type strain. AlkB expression was pre-induced with DCPK, and wells were oxygenated and saturated with excess octane substrate $(30 \% \mathrm{v} / \mathrm{v})$

range construct. These results support the knockout assay demonstrating that FadL, rather than OmpW, serves as the major route for octane uptake in E. coli (Fig. 3). The greatest activity, not surprisingly, was observed with the natural alkane importer, AlkL, at $1000 \mu \mathrm{M}$ L-rhamnose, resulting in a maximum conversion rate of $119 \mu \mathrm{mol} \mathrm{min}{ }^{-1} \mathrm{~g}^{-1}$.

Although the tighter rhaBAD promoter may have helped to resolve leaky or poorly controlled expression issues, intrinsic differences in induction, folding and translocation efficiencies may still have impacted optimal inducer concentration. Furthermore, subtle differences in the affinity of AlkL OmpW, and FadL for octane could also account for the variations in optimal inducer concentrations. Nonetheless, it is clear that optimizing import to maximize conversion rates of octane by AlkBGT not only requires choosing the right expression system, but also sensitive tuning of induction levels.

\section{Removal of fadL confers tolerance toward medium chain alkanes}

Previously, medium chain alkanes have been reported to exert toxic growth effects [21-23]. In particular, solvents with octanol-water phase partition coefficients $\left(\log P_{o w}\right)$ between 1.5 and 6.0 are known to alter membrane structure and interfere with respiratory ATP metabolism [24]. Having identified FadL as a significant mediator of octane import, we hypothesized that removal of the import route, through deletion of FadL, may improve tolerance toward medium-chain alkanes such as hexane and octane which have $\log P_{o w}$ values of 3.8 and 4.8, respectively. The cell growths of the wild type, $\Delta f a d L$ and $\triangle o m p W$ strains were therefore assessed by monitoring $\mathrm{OD}_{600}$ in the presence and absence of hexane and octane. The $\Delta$ marR strain was included as a positive control since previous studies had shown it to confer solvent resistance to alkanes including hexane [21, 25, 26]. Growth rates in the absence of solvents were found to be similar, no significant differences in $\mathrm{OD}_{600}$ values were noted between all the strains (Additional file 1: Figure S3). However, in the presence of $10 \%(\mathrm{v} / \mathrm{v})$ hexane, a clear decrease in cell density was observed for the control and $\triangle o m p W$ strains suggesting loss of cell viability, whereas $\triangle f a d L$ strain neither increased nor decreased in cell density indicating that viability in this particular strain could be maintained (Fig. 5). In contrast and in accordance with previous studies, the $\triangle$ marR strain was capable of further growth. A similar observation was also made under conditions using $10 \%(\mathrm{v} / \mathrm{v})$ octane though the effect was less pronounced, presumably due to the lower cellular toxicity of octane in comparison to hexane (Additional file 1: Figure S4). ANOVA was applied to the dataset to test for statistical significance (Additional file 1: Figure S3). Based on differences in OD values and specific growth rates, a significant interaction between the presence of alkane and the FadL gene knockout ( $F$ values of 16.26 and 10.42; $p$-values of 0.0004 and 0.002 ) was observed while differences

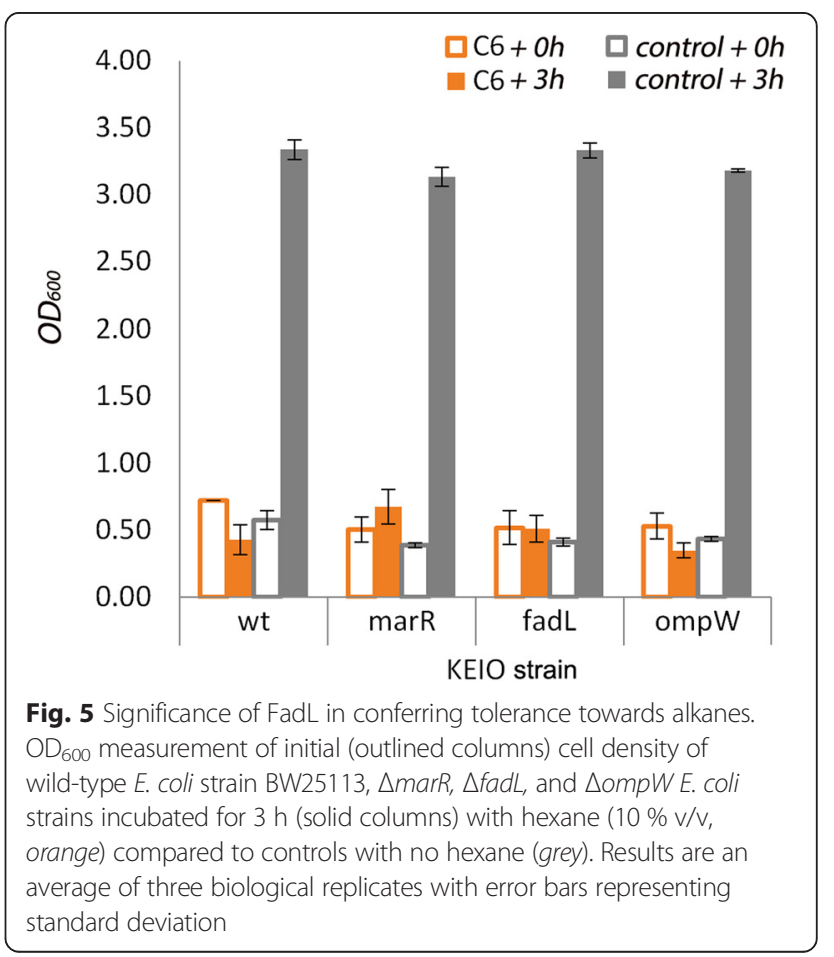


between replicates were found to be insignificant ( $p$-value of 0.58 and 0.78 ).

Given that the viability of $E$. coli in the presence of high alkane concentration is less affected in a $\Delta f a d L$ genetic background suggests that tolerance could be engineered by reducing or abolishing FadL activity. Such an approach would reduce toxicity by effectively lowering the rate of intracellular influx of alkanes. For the $\triangle$ marR strain, which was found to possess greater growth resilience in the presence of alkanes, the physiological explanation is probably somewhat more complex since multiple stress-related mechanisms are most likely to operate simultaneously [22]. The induction of the multi-drug efflux pump, AcrAB-TolC, is one such response which is known to confer resistance to multiple drugs, in addition to alkanes including octane [23]. It remains possible that, during the course of this study, the native house-keeping activity of the AcrAB-TolC complex may well have partially masked the tolerance benefit imparted by the deletion of FadL. In light of this, AcrAB-TolC mutants may provide a more ideal genetic background for evaluating the significance of FadL in conferring tolerance toward a range of chemicals including alkanes.

\section{Impact of native alkane import on whole-cell alkane biosensing}

In a previous study, we had assembled a whole-cell biosensing system for alkanes by coupling the alkanebinding transcriptional regulator, AlkS from the $P$.

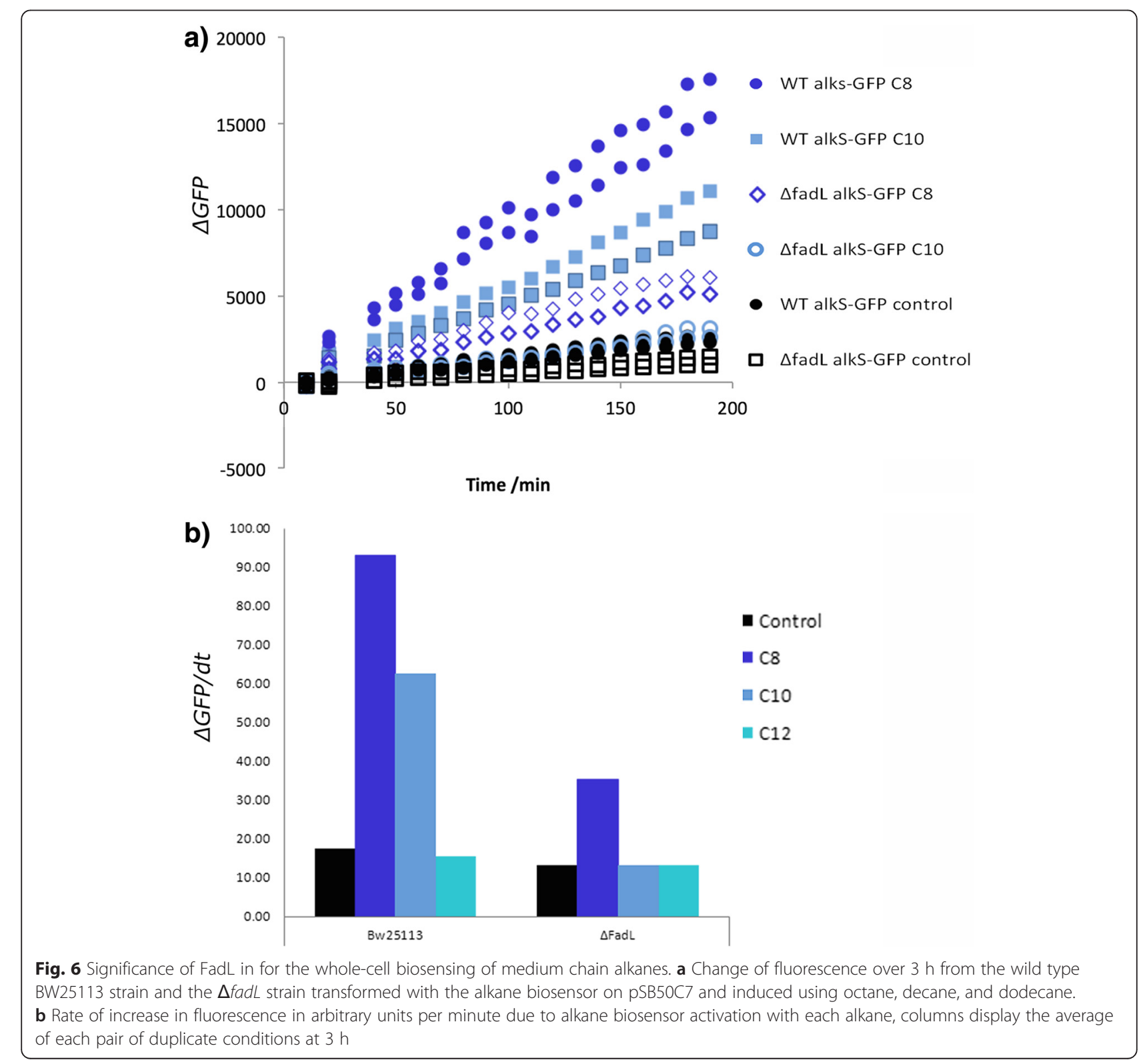


putida alkane degradation pathway [24, 27], to a GFP reporter protein and had shown that the signal response for intracellular alkane biosensing could be markedly improved by increasing the expression levels of the AlkL importer. On this basis, we reasoned that if FadL facilitated the import of alkanes then a drop in signal intensity would be expected in a $\Delta f a d L$ background. To experimentally verify this, the AlkS/GFP-based sensor built previously [4] was transferred to both parental $E$. coli BW25113 and $\Delta f a d L$ strains harbouring the pSB50C7 plasmid, and fluorescence was monitored in the presence of octane, decane, and dodecane. As predicted, the fluorescence signal (expressed in arbitrary units) of the $\Delta f a d L$ strain, compared to the parental strain, decreased by 2.7- and 2.9- fold in the presence of octane and decane respectively, thus corroborating the hypothesis that FadL can mediate the import of medium chain alkanes (Fig. 6a and b). By contrast and as expected, there was no significant fluorescence decrease in the presence of dodecane since AlkS acts specifically on C6-C11 alkanes [24, 27]. Further work to engineer an intracellular alkane biosensor with broader specificity could be used to investigate and extend the range of chain length specificity of FadL alkane import.

Controlling the import of signal-eliciting molecules will be important for the development of advanced genetic circuits (including biosensing systems) that respond to external stimuli in a precise, predictable and dosedependent manner [28]. In this regard, transmembrane importers such as FadL could serve as critical targets for engineering purposes given their importance in influencing the signal response of whole-cell biosensing systems.

\section{Conclusion}

In conclusion we show FadL to be the principle route for octane import in E. coli. This study provides a useful toolkit of experimental approaches for evaluating alkane import, and highlights the importance of native outer membrane importers such as FadL for the purpose of developing and optimizing biological applications such as bioremediation and biofuels.

\section{Methods}

\section{Bioinformatics}

Initial identification of candidate genes was expanded from the literature search using online resources: Ecocyc database, (http://www.ncbi.nlm.nih.gov/pmc/articles/ PMC99147/). OmpW was previously identified as a target via BLAST searching, revealing identity with the known alkane importer AlkL [4]. Structural alignment and viewing of FadL, AlkL and OmpW was performed using Pymol. The AlkL models were generated using Swiss Model [29, 30], using the 2f1t $x$-ray crystal structure as a template. Membrane topology was predicted using the orientations of proteins in membranes database [31].

\section{Strains}

The Keio collection, a knockout library of all nonessential genes in E. coli [19], and ASKA collection, a clonal plasmid library of all individual $E$. coli gene open reading frames [32] were obtained from the NBRP, Nara University of Science and Technology. Both collections are derived from the E. coli $\mathrm{K}-12$ strain and allow systematic studies of gene function based on gene overexpression or complementation. The Keio strains have each gene replaced on the chromosome by a kanamycin resistance gene flanked by FRT recombination sites for removal if necessary. The ASKA strains harbor plasmids with the open reading frame (ORF) for each gene, expressed under control of the LacIa promoter, and a chloramphenicol resistance marker. See Additional file 1: Table S1 for a list of all strains used in this study.

\section{Plasmid construction}

The pGEc41 plasmid is $51.6 \mathrm{~kb}$ in size and is constructed from a $21 \mathrm{~kb}$ vector pLAFR1 containing a $30 \mathrm{~kb}$ fragment with alk operon genes alkBFGHJKL and alkST from Pseudomonas putida with the omission of alkJ (an alcohol dehydrogenase), alkK (an acyl-CoA synthetase) and alkL [33]. Gene induction was placed under the control of the AlkS - pAlkB promoter system. ASKA plasmids containing the ORF for each target gene were isolated from the ASKA collection [32]. They contain ORFs for all E. coli genes cloned between Sfil restriction sites, and under the control of isopropyl- $\beta$-D-thiogalactopyranoside (IPTG) inducible lac1q promoter. See Additional file 1: Table S1 for a list of all plasmids used in this study.

\section{Media preparation}

Lysogeny Broth (LB) growth medium; composed of yeast extract $10 \mathrm{~g} / \mathrm{L}$, tryptone $20 \mathrm{~g} / \mathrm{L}$ and $\mathrm{NaCl} 20 \mathrm{~g} / \mathrm{L}$; was used for most overnight inoculations, Octane uptake and conversion assays were performed in 'Wubbolts' enhanced conversion media, taken from Wubbolts et al. [34]. The composition of Wubbolts medium is as follows: $\mathrm{KH}_{2} \mathrm{PO}_{4}, 4 \mathrm{~g} / \mathrm{L} ; \mathrm{K}_{2} \mathrm{HPO}_{4}\left(3 \mathrm{H}_{2} \mathrm{O}\right), 15.9 \mathrm{~g} / \mathrm{L}$; $\mathrm{Na}_{2} \mathrm{HPO}_{4}\left(12 \mathrm{H}_{2} \mathrm{O}\right), 7 \mathrm{~g} / \mathrm{L} ;\left(\mathrm{NH}_{4}\right)_{2} \mathrm{SO}_{4}, 1.2 \mathrm{~g} / \mathrm{L} ; \mathrm{NH}_{4} \mathrm{Cl}$, $0.2 \mathrm{~g} / \mathrm{L}$ (all from Sigma Aldrich); yeast extract, $5 \mathrm{~g} / \mathrm{L}$; Lleucine, $0.6 \mathrm{~g} / \mathrm{L}$; L-proline, $0.6 \mathrm{~g} / \mathrm{L}$; thiamine, $5 \mathrm{mg} / \mathrm{L}$. The following components were autoclaved and added separatelyo add post autoclaving: $\mathrm{MgSO}_{4}\left(7 \mathrm{H}_{2} \mathrm{O}\right)(\mathrm{BDH})$, $1 \mathrm{~g} / \mathrm{L}(\mathrm{BDH}) ; 1 \mathrm{ml}$ of trace minerals (composition below); $1 \mathrm{ml}$ of $4 \%$ (w/v) $\mathrm{CaCl}_{2}\left(2 \mathrm{H}_{2} \mathrm{O}\right)$ (Alfa Aesar) and $10 \mathrm{~g} / \mathrm{L}$ D-glucose, were added having all been heat sterilized separately. Filter- sterilized antibiotics were added 
as appropriate. The trace minerals solution contained per litre of $5 \mathrm{M} \mathrm{HClL}: 40 \mathrm{~g} \mathrm{FeSO}_{4}\left(7 \mathrm{H}_{2} \mathrm{O}\right), 10 \mathrm{~g}$ $\mathrm{MnSO}_{4}\left(\mathrm{H}_{2} \mathrm{O}\right), 4.75$ g $\mathrm{CoCl}_{2}\left(6 \mathrm{H}_{2} \mathrm{O}\right), 2 \mathrm{~g} \mathrm{ZnSO}_{4}$ $\left(7 \mathrm{H}_{2} \mathrm{O}\right), 2$ g $\mathrm{MoO}_{4} \mathrm{Na}_{2}\left(2 \mathrm{H}_{2} \mathrm{O}\right), 1 \mathrm{~g} \mathrm{CuCl},\left(2 \mathrm{H}_{2} \mathrm{O}\right)$ and, $0.5 \mathrm{~g} \mathrm{H}_{3} \mathrm{BO}_{3}$. Antibiotics were used at the following working concentrations: tetracycline $(10 \mathrm{mg} /$ $\mathrm{L})$, chloramphenicol $(25 \mathrm{mg} / \mathrm{L})$, Zeocin $(100 \mathrm{mg} / \mathrm{L})$ and kanamycin $(30 \mathrm{mg} / \mathrm{L})$.

\section{In-vivo conversion of octane}

Comparative bioconversion assays were carried out in 24 deep-well Teflon plates to reduce the adsorption of product and substrate on the on the walls of individual wells. Strains being tested were inoculated overnight in $5 \mathrm{ml} \mathrm{LB}$ in $50 \mathrm{ml}$ Falcon tubes with relevant antibiotics for all strains and plasmids. At least 3 biological repeats were isolated from the original transformation plate for each plasmid and condition tested. $1 \mathrm{ml}$ culture volumes were transferred to each well accordingly, and $300 \mu \mathrm{l}$ of octane (Sigma-Aldrich) was added at $t=0$. The initial assay (Fig. 3) was carried out in LB media in plates sealed with aluminium foil and clamped with a film cover (EnzyScreen) to counter octane evaporation, and without pre-induction of pGEc41. For overexpression of omp W and $f a d L$, appropriate concentrations of IPTG were added prior to overnight culture and octane addition. For later assays (Additional file 1: Figure S2 and Fig. 4), $300 \mu$ l of substrate was deemed sufficient to counter the effects of evaporation and ensure that substrate availability was not rate-limiting. Gas transfer was facilitated, via a pinhole in the sandwich cover. For preinduction of pGEc41 in Additional file 1: Figure S2 and Fig. 4, strains were sub-cultured 1:20 in $1 \mathrm{ml}$ of enhanced conversion media (Wubbolt's media) for $1 \mathrm{~h}$ and then induced with $0.05 \%(\mathrm{w} / \mathrm{v})$ Dicyclopropylketone (DCPK) and incubated at $37^{\circ} \mathrm{C}$ for a further $4 \mathrm{~h}$. For expression from the pRHA67k-derived plasmids, Lrhamnose was added after $1 \mathrm{~h}$ of growth in Wubbolt's media and $4 \mathrm{~h}$ prior to octane addition. Assay plates were clamped shut in the shaker incubator (Kuhner) at $37{ }^{\circ} \mathrm{C}$ and shaken at 250 RPM with a throw diameter of $25 \mathrm{~mm}$. Well contents were harvested after $8 \mathrm{~h}$ (Fig. 3 and Additional file 1: Figure S1) and $1 \mathrm{~h}$ (Fig. 4 and Additional file 1: Figure S2) and transferred to preweighed $2 \mathrm{ml}$ Eppendorf tubes, centrifuged at 13,000 RPM for $5 \mathrm{~min}$ and the supernatant poured into separate tubes. After addition of $800 \mu$ l ethyl acetate (Sigma-Aldrich) to separate tubes containing the supernatant and cell pellet, each was vortexed for 1 min to separate the alkanes along with the alkane-derived oxidized products, and then centrifuged for $1 \mathrm{~min}$ to separate the aqueous and organic phases. A $600 \mu \mathrm{l}$ volume of the top phase (organic phase) was transferred to glass vials for analysis by GC-FID.

\section{Tolerance assays}

Seed cultures of the strains were grown overnight in 96well DSW plates and sub-cultured (using a $5 \%(\mathrm{v} / \mathrm{v})$ inoculum) into fresh LB media at $37{ }^{\circ} \mathrm{C}$, shaking at $250 \mathrm{rpm}$ (25 mm amplitude) with absorbance readings monitored at $600 \mathrm{~nm}$ using a BMG Clariostar plate reader after $0 \mathrm{~h}$ and $2 \mathrm{~h}$. Half the cultures were spiked with an excess of $10 \% \mathrm{v} / \mathrm{v} n$-alkane and incubated for a further $3 \mathrm{~h}$ until the alkane had evaporated, after which point a final set of OD readings was taken.

\section{Metabolite analysis}

Determination of alkanes and their respective oxidized products in microwells was achieved via sacrificial sampling and extraction of both phases into ethyl acetate directly. $800 \mu \mathrm{l}$ of ethyl acetate was added to the two-phase supernatant following centrifugation at 13,000 rpm for $5 \mathrm{~min}$ in a microfuge. The samples were vortexed for $3 \times 20 \mathrm{~s}$, prior to centrifugation for $1 \mathrm{~min}$ and removal of $100 \mu \mathrm{l}$ of the organic phase, and then analysed by GC-FID using a Perkin Elmer Autosystem XL equipped with a SGE BPX5 (30 m long; $0.53 \mathrm{~mm}$ internal diameter, $1 \mu \mathrm{m}$ film) capillary column and helium as the carrier gas under a constant pressure of 4 PSI. The proportion of organic phase was determined by cross-referencing the $n$-alkane peak size to a calibration curve of known standards. Alkanes along with their oxidized derivatives were separated and quantified using the following GC-FID method. For n-octane oxidation product determination the samples were eluted at $70^{\circ}$ $\mathrm{C}$ followed by a linear increase of $5{ }^{\circ} \mathrm{C}$ minute $e^{-1}$ to reach a final temperature of $145^{\circ} \mathrm{C}$. All standards were purchased from Alfa Aesar at the highest purity available (>98\%).

\section{Determination of dry cell weight}

Cell density measurements were taken by sacrificing the well contents and centrifuging the biphasic samples at $13,000 \mathrm{rpm}(19,000 \mathrm{~g})$ for $5 \mathrm{~min}$, marking the aqueous volume on the side of the graduated Eppendorf tube; pellets were washed with Tris- $\mathrm{HCl} \mathrm{pH7.4} \mathrm{and} \mathrm{dried} \mathrm{in}$ an $80{ }^{\circ} \mathrm{C}$ oven until a constant mass was reached.

\section{Alkane biosensor assay}

E. coli BW25113 and the Keio $\Delta f a d L$ strain were transformed with an alkS-Superfolder GFP biosensor on pSB50C7. The strains were grown up overnight from single colonies in parallel in deep square well plates ( $1 \mathrm{ml}$ per well) at $37^{\circ} \mathrm{C}$ and $250 \mathrm{rpm}$ ( $25 \mathrm{~mm}$ amplitude) in Luria broth containing $5 \mathrm{~g} / \mathrm{L}$ glucose and then sub cultured ( $4 \%$ incoculum) with $10 \mathrm{~g} / \mathrm{L}$ glycerol added, grown for $2 \mathrm{~h}$, and induced using $3 \mathrm{mM}$-arabinose. After $3 \mathrm{~h}$ growth, $1 \% \mathrm{v} / \mathrm{v}$ alkane substrates were added and grown for $3 \mathrm{~h}$, analysing fluorescence every $10 \mathrm{~min}$ (excita- tion $485 \mathrm{~nm}$; emission $517 \mathrm{~nm}$ ) using a BMG Clariostar plate reader. 


\section{Additional file}

Additional file 1: Supplementary Information [35, 36]. (DOCX 25764 kb)

\section{Competing interests}

The authors declare that they have no competing interests.

\section{Authors' contributions}

TC performed the knockout, complementation, and IPTG induction bioconversion assays. CG constructed the rhamnose inducible vector and performed the rhamnose induction assays and biosensor experiments and designed the study. TC and CG, drafted the manuscript, with significant contributions from MKA and FB. All authors have read and approved the final manuscript.

\section{Acknowledgements}

The authors would like to acknowledge the Engineering and Physical Sciences Research Council (EPSRC) and BBSRC for a Masters training grant $\mathrm{BB} / \mathrm{H} 021027 / 1$ for funding. The research was performed at the Advanced Centre for Biochemical Engineering, University College London. The authors would like to acknowledge the NBRP-E.coli at NIG for the KEIO and ASKA collections and Adam Denyer who performed some of the work leading to the toxicity study.

\section{Supporting Information Available}

Including bioconversion assay data at varying inducer concentrations and western blot data. This material is available free of charge via the Internet at http://pubs.acs.org.

\section{Author details}

'Department of Biochemical Engineering, Advanced Centre for Biochemical Engineering, University College London, Torrington Place, London WC1E 7JE, UK. ${ }^{2}$ Present address: Department of Biochemistry, University of Cambridge, 80 Tennis Court Road, Cambridge CB2 1GA, UK. ${ }^{3}$ Present address: Institute of Molecular Plant Sciences, School of Biological Sciences, University of Edinburgh, Edinburgh EH9 3BF, UK.

\section{Received: 23 February 2016 Accepted: 29 March 2016}

\section{Published online: 05 April 2016}

\section{References}

1. Wilcox J. Grand challenges in advanced fossil fuel technologies. Front Energy Res. 2014;2(47):1-3

2. Zhu L, Zhu Y, Zhang Y, Li Y. Engineering the robustness of industrial microbes through synthetic biology. Trends Microbiol. 2012;20(2):94-101.

3. Moldes AB, Paradelo R, Rubinos D, Devesa-Rey R, Cruz JM, Barral MT. Ex situ treatment of hydrocarbon-contaminated soil using biosurfactants from lactobacillus pentosus. J Agric Food Chem. 2011;59(17):9443-7.

4. Grant C, Deszcz D, Wei Y-C, Martínez-Torres RJ, Morris P, Folliard T, Sreenivasan R, Ward J, Dalby P, Woodley JM, Baganz F. Identification and use of an alkane transporter plug-in for applications in biocatalysis and whole-cell biosensing of alkanes. Sci Rep. 2014;4:5844.

5. Choi YJ, Lee SY. Microbial production of short-chain alkanes. Nature. 2013; 502(7472):571-4

6. Julsing MK, Schrewe M, Cornelissen S, Hermann I, Schmid A, Bühler B. Outer membrane protein AlkL boosts biocatalytic oxyfunctionalization of hydrophobic substrates in Escherichia coli. Appl Environ Microbiol. 2012; 78(16):5724-33.

7. Rojo F. Specificity at the end of the tunnel: understanding substrate length discrimination by the AlkB Alkane hydroxylase. J Bacteriol. 2005;187(1):19-22.

8. Favre-Bulle O, Weenik E, Vos T, Preusting H, Witholt B. Continuous bioconversion of $n$-octane to octanoic acid by recombinant Escherichia coli (alk(+)) growing in a two-liquid-phase Chemostat. Biotechnol Bioeng. 1993; 41(2):263-72.

9. Ji Y, Mao G, Wang Y, Bartlam M. Structural insights into diversity and n-alkane biodegradation mechanisms of alkane hydroxylases. Front Microbiol. 2013:4:58

10. Eggink G, Lageveen RG, Altenburg B, Witholt B. Controlled and functional expression of the Pseudomonas oleovorans alkane utilizing system in Pseudomonas putida and Escherichia coli. J Biol Chem. 1987;262(36):17712-8.
11. Favre-Bulle $\mathrm{O}$, Schouten $T$, Kingma J, Witholt B. Bioconversion of n-octane to octanoic acid by a recombinant Escherichia coli cultured in a two-liquid phase bioreactor. Nat Biotechnol. 1991;9:367-71.

12. Black PN. Characterization of FadL-specific fatty acid binding in E. coli. Biochim Biophys Acta. 1990;1046(1):97-105.

13. Nunn WD, Simons RW. Transport of long-chain fatty acids by Escherichid coli: mapping and characterization of mutants in the fadL gene. Proc Natl Acad Sci U S A. 1978;75(7):3377-81.

14. van den Berg B. Going forward laterally: transmembrane passage of hydrophobic molecules through protein channel walls. Chembiochem. 2010;11(10):1339-43.

15. Hong H, Patel DR, Tamm LK, van den Berg B. The outer membrane protein OmpW forms an eight-stranded beta-barrel with a hydrophobic channel. J Biol Chem. 2006;281(11):7568-77.

16. Lepore BW, Indic M, Pham H, Hearn EM, Patel DR, van den Berg B. Ligandgated diffusion across the bacterial outer membrane. Proc Natl Acad Sci U S A. 2011;108(25):10121-6.

17. Van den Berg B. The FadL family: unusual transporters for unusual substrates. Curr Opin Struct Biol. 2005;15(4):401-7.

18. Simard JR, Pillai BK, Hamilton JA. Fatty acid flip-flop in a model membrane is faster than desorption into the aqueous phase. Biochemistry. 2008;47(35):9081-9.

19. Baba T, Ara T, Hasegawa M, Takai Y, Okumura Y, Baba M, Mori H. Construction of Escherichia coli K-12 in-frame, single-gene knockout mutants: the Keio collection. Mol Syst Biol. 2006;2:2006.0008.

20. Black PN, Zhang Q. Evidence that His 110 of the protein FadL in the outer membrane of Escherichia coli is involved in the binding and uptake of longchain fatty acids: possible role of this residue in carboxylate binding. The Biochemical Journal. 1995;310(Pt 2):389-94.

21. Aono R. Improvement of organic solvent tolerance level of Escherichia coli by overexpression of stress-responsive genes. Extremophiles. 1998;2(3):239-48.

22. Diaz E. Microbial degradation: Genomics and Molecular Biology. Caister Academic Press; 2008

23. Foo JL, Leong SSJ. Directed evolution of an E. coli inner membrane transporter for improved efflux of biofuel molecules. Biotechnol Biofuels. 2013;6(1):81.

24. Reed B, Blazeck J, Alper $\mathrm{H}$. Evolution of an alkane-inducible biosensor for increased responsiveness to short-chain alkanes. J Biotechnol. 2012;158(3):75-9.

25. Collao B, Morales EH, Gil F, Calderón IL, Saavedra CP. OmpW is cooperatively upregulated by MarA and SoxS in response to menadione. Microbiology. 2013;159:715-25.

26. Doukyu N, Ishikawa K, Watanabe R, Ogino H. Improvement in organic solvent tolerance by double disruptions of proV and marR genes in Escherichia coli. J Appl Microbiol. 2012;112(3):464-74.

27. Canosa I, Sánchez-Romero JM, Yuste L, Rojo F. A positive feedback mechanism controls expression of AlkS, the transcriptional regulator of the Pseudomonas oleovorans alkane degradation pathway. Mol Microbiol. 2000; 35(4):791-9.

28. Zhang F, Carothers JM, Keasling JD. Design of a dynamic sensor-regulator system for production of chemicals and fuels derived from fatty acids. Nat Biotechnol. 2012;30(4):354-9.

29. Kiefer F, Arnold K, Künzli M, Bordoli L, Schwede T. The SWISS-MODEL Repository and associated resources. Nucleic Acids Res. 2009;37:D387.

30. Kopp J, Schwede T. The SWISS-MODEL Repository of annotated threedimensional protein structure homology models. Nucleic Acids Res. 2004;32: D230.

31. Lomize MA, Lomize AL, Pogozheva ID, Mosberg HI. OPM:orientations of proteins in membranes database. Bioinforma. 2006;22:623-5.

32. Kitagawa M, Ara T, Arifuzzaman M, Loka-nakamichi T. Complete set of ORF clones of Escherichia coli ASKA library (A Complete Set of E.coli K-12 ORF Archive): Unique Resources for Biological Research. DNA Res. 2006:12:291-9.

33. Austin RN, Chang HK, Zylstra GJ, Groves JT. The non-heme diiron alkane monooxygenase of Pseudomonas oleovorans (AlkB) hydroxylates via a substrate radical intermediate. J Am Chem Soc. 2000;122(47):11747-8.

34. Wubbolts MG, Favre-Bulle $\mathrm{O}$, Witholt B. Biosynthesis of synthons in twoliquid-phase media. Biotechnol Bioeng. 1996;52(2):301-8.

35. Boyer HW, Roulland-Dussoix DJ. Mol Biol. 1969:41:459-72.

36. Giacalone MJ, Gentile AM, Lovitt BT, Berkley NL, Gunderson CW, Surber MW. Toxic protein expression in Escherichia coli using a rhamnose-based tightly regulated and tunable promoter system. BioTechniques. 2006:40:355-63. 